\title{
A great mimicker of primary biliary cholangitis
}

\author{
B. Zwaenepoel ${ }^{1}$, E. Padalko², A. Hoorens ${ }^{3}$, S. Vanooteghem ${ }^{4}$, P. Hindryckx ${ }^{1}$
}

(1) Division of gastroenterology and hepatology, Ghent University Hospital, Belgium; (2) Laboratory of medical microbiology, Ghent University Hospital, Belgium; (3) Division of pathology, Ghent University Hospital, Belgium; (4) Division of gastroenterology and hepatology, Waregem Regional Hospital, Belgium.

\section{Introduction}

A 59 year-old man without past medical history was referred with biochemical features of cholestasis (aspartate aminotransferase (AST) $117 \mathrm{U} / \mathrm{L}$, alanine aminotransferase (ALT) $83 \mathrm{U} / \mathrm{L}$, gamma-glutamyl transferase (GGT) 1307 U/L, alkaline phosphatase (AP) 1803 $\mathrm{U} / \mathrm{L}$, total bilirubin $0,7 \mathrm{mg} / \mathrm{dL}$ ), a strongly positive antimitochondrial M2 antibody (AMA-M2) titer (88 U), weight loss and abdominal pain since several months. He did not take any medications, nor there was a history of alcohol abuse or sexual risk behavior. Upon presentation, clinical examination showed a rather cachectic patient (body mass index $19 \mathrm{~kg} / \mathrm{m}^{2}$ ), without further abnormalities. As primary biliary cholangitis (PBC) was suspected, treatment with ursodeoxycholic acid had been initiated but did not improve the cholestasis nor the pain. Additional investigations didn't reveal any other irregularities, except for a mildly dilated aortic sinus root of $42 \mathrm{~mm}$. The histopathological findings of a liver biopsy are shown below (Figure 1). This showed granulomas with cholangitis and increased presence of neutrophils, which raised suspicion for an infectious cause.

\section{Question}

Which infectious agent is re-emerging in human society and can mimic PBC?

\section{Answer}

Additional serology revealed a strongly reactive treponemal antibody test (TT) (signal-to-cutoff ratio 18,77), a negative rapid plasma regain test (RPR) and a reactive confirmatory TT. The patient was treated with benzathine benzylpenicillin 2,4 MIU given at weekly intervals during three weeks, which resulted in quick resolution of the long-lasting pain and significant reduction of liver biochemistry (AST $41 \mathrm{U} / \mathrm{L}$, ALT $31 \mathrm{U} / \mathrm{L}$, GGT $427 \mathrm{U} / \mathrm{L}$, AP $881 \mathrm{U} / \mathrm{L}$, total bilirubin $0,6 \mathrm{mg} / \mathrm{dL}$ on day 60 after treatment). Clinical improvement combined with serology and pathology lead to the final diagnosis of tertiary syphilis with hepatic involvement.

Syphilis is currently re-emerging at high speed (1). Liver involvement is rather uncommon and frequently overlooked as a cause of liver function abnormalities. The differential diagnosis with other causes of cholestatic liver injury is challenging as symptoms and signs are often non-specific (2). The presence of AMA-M2, usually

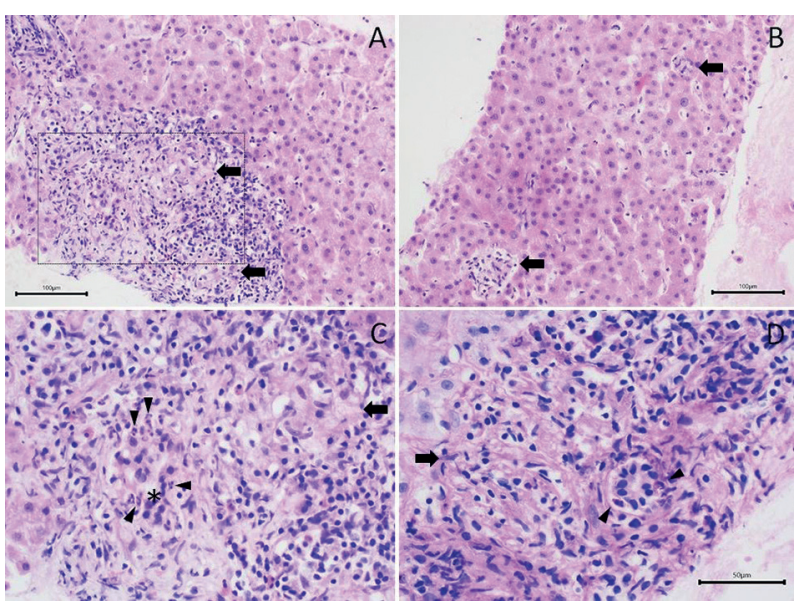

Figure 1. - Liver biopsy: A. Portal tract inflammation with lymphocytes, rare plasma cells, several eosinophils and neutrophils and non-necrotizing epithelioid granulomas (arrows) and very scant lobular lymphocytic infiltration (hematoxylin and eosin stain (H\&E)). B. Lobular micro-granulomas (arrows) (H\&E). C. (Higher magnification of inset in A) Portal tract with granuloma (arrow) and bile duct (asterisk in center of bile duct) with slightly damaged epithelium and several neutrophils (arrowheads) surrounding cholangiocytes (H\&E). D. Portal tract with granuloma and bile duct (arrow) with sparse neutrophils located within the basement membrane (PAS-diastase).

highly specific for $\mathrm{PBC}$, can be positive due to mimicry (3). A reactive TT is considered necessary for diagnosis, whereas a RPR can be non-reactive in long-standing untreated disease (1). Rapid remission of clinical and biochemical hallmarks after treatment is considered an important additional diagnostic clue (2).

\section{Conflicts of Interest}

The authors declare no conflict of interest.

\section{References}

1. GHANEM KG, RAM S, RICE PA. The modern epidemic of syphilis. $N$ Engl JMed., 2020, 382: 845-54.

2. MULLICK CJ, LIAPPIS AP, BENATOR DA, ROBERTS AD, PARENTI DM, SIMON GL. Syphilitic Hepatitis in HIV-Infected Patients: A Report of 7 Cases and Review of the Literature. Clin Infect Dis., 2004, 39: e100-5.

3. HORN CL, JALALI S, ABBOTT J, STEIN MT. A Surprising Diagnosis: Syphilitic Gastritis and Hepatitis. Am J Med., 2018, 131: 1178-81.

Correspondence to : Bert Zwaenepoel, M.D., Division of gastroenterology and hepatology, University Hospital Ghent, Corneel Heymanslaan, 10, 9000 Ghent, Belgium. Fax: +3293323800.

Email: bert.zwaenepoel@uzgent.be

Submission date : 16/12/2020

Acceptance date : 14/01/2021 